\title{
The Past, Present and Future of Enzyme Measurements Using Surface Enhanced Raman Spectroscopy (SERS)
}

\author{
Iain A. Larmour, Karen Faulds and Duncan Graham* \\ Received (in XXX, XXX) Xth XXXXXXXXX 200X, Accepted Xth XXXXXXXXX 200X \\ ${ }_{5}$ First published on the web Xth $X X X X X X X X X 200 X$ \\ DOI: 10.1039/b000000x
}

The ability to accurately and sensitively measure the activity of specific enzymes is central to many aspects of modern chemistry and when combined with new nanoscience based approaches, offers significant opportunities for advancing other scientific disciplines. We review the 10 development of surface enhanced resonance Raman scattering (SERRS) for the detection of enzymes, from the initial direct spectroscopy of enzymes, substrate/product and inhibitors adsorbed onto metallic colloids, to the current approach of measuring enzymatic activity by recording the SERRS spectra of a product which is only 'switched on' after enzyme activity. Developments focussed on improvements to modular masked SERRS substrates, which are 15 unmasked by specific enzymes, are also reviewed. Finally, we set out the remaining grand challenges within the area of enzymatic analysis by SERRS which include single molecule detection, in vivo studies and increased multiplexing for screening of evolved enzyme libraries.

\section{Introduction}

DNA may carry the blueprint of life from one generation to 20 another, however it is the proteins which are encoded for within our DNA, that are responsible for almost every process that occurs during an organism's life. Proteins are involved in genetic regulation, defence, transportation as well as in the structural integrity of an organism. ${ }^{1}$ In this review we 25 concentrate on the workhorses of the protein world, enzymes.

Of the 63,000 protein structures currently deposited in the protein data bank, 11,900 structures are enzymes. Enzymes are involved in a myriad of processes from signal transduction, muscle contraction and digestion ${ }^{2}$ to DNA 30 processes $^{3,4}$ and are broadly classified by their main function; oxidoreductases, transferases, hydrolases, lyases, isomerases and ligases. Without enzymes many of the essential biological reactions would occur at a rate that could not support life. Enzymes can enhance reaction rates by up to $10^{15}$ times and 35 turnover millions of substrates per second. ${ }^{5}$ In addition to their rate enhancing ability, they are extremely selective which has made them ideal for use within many industrial sectors such as; food processing, biofuel production and in biological detergents. ${ }^{2,6}$ It is these pivotal roles that enzymes 40 play that make them such essential molecules to study. This review focusses on the use of surface enhanced (resonance) Raman scattering, SE(R)RS to study enzymes, their activity and their use in assays..

The discovery of the surface enhanced Raman scattering 45 (SERS) effect by Fleischmann et al. in $1974^{7}$ gave the field of Raman spectroscopy a new lease of life. Previously, Raman had suffered due to its inherently weak signal, however Fleischmann showed that by the use of an appropriately roughened metallic substrate the signal could be increased by $50 \sim 10^{6}$. If the molecule under investigation also has an electronic absorption close to the laser excitation wavelength this can give rise to resonance enhancements of the order of $10^{3}-10^{4} .8,9$ Coupled with SERS this results in surface enhanced resonance Raman scattering (SERRS) which has an 55 overall enhancement potential of $10^{10}$, although enhancements of up to $10^{14}$ have been reported. ${ }^{10,11}$ Advances in nanotechnology techniques have opened up a range of novel and reproducible substrates which give high enhancements ideal for SE(R)RS. ${ }^{12-14}$ In addition to advances made in 60 substrate development, the range of applications for SE(R)RS is rapidly expanding. ${ }^{15-18}$

The use of SE(R)RS for biomedical applications has three significant features which make it an ideal technique. Firstly, the significantly enhanced signals which are possible make it 65 ideal for dilute solutions. Secondly, complex biological fluids tend to give significant levels of background fluorescence and metallic substrates used for SE(R)RS have the ability to quench this fluorescence. Thirdly, SERS is a surface selective technique, the enhancement quickly diminishes with the 70 distance of the analyte from the surface, therefore, only the species that are in close proximity to the surface will be enhanced, allowing selective detection of a specific analyte from a complex matrix. ${ }^{19}$ When compared to fluorescence, SERRS also has significant benefits which include sensitivity 75 and abundant, sharp and well separated bands due to the fingerprint spectrum obtained, which is ideal for multiplexing. ${ }^{20-22}$ Reporter molecules, such as a coloured dye molecule, do not self-quench therefore, the signal can be increased by increasing the number of reporters and unlike 80 optical absorption techniques the signal cannot over develop. ${ }^{23}$

This review comprises of four main areas; initially we look at SE(R)RS studies of enzymes, coenzymes and enzyme substrates directly coupled to the enhancing medium before 85 reviewing work where the signal is produced from a dye created by the action of an enzyme. Then we explore signal generation from a dye that is unmasked by an enzyme, before 
discussing the future challenges and directions within the field.

\section{Direct Enzyme SE(R)RS}

Studies of enzyme substrates ${ }^{24-26}$ and coenzymes ${ }^{27-29}$ ${ }_{5}$ immobilised on silver or gold electrodes have been reported where the spectral changes of these moieties can be monitored either with variation of the electrode potential or by the addition of an enzyme. This gives information on the effect an enzyme has on these species but gives minimal information 10 about the enzyme itself.

SE(R)RS obtained directly from enzymes has been investigated for over 20 years. One of the first reports was by Copeland et al. where the SERRS of a glucose oxidase was reported on colloidal silver nanoparticles. ${ }^{30} \mathrm{~A}$ high-quality 15 flavin spectrum was recorded due to the fluorescence quenching provided by the metallic nanoparticles. There was a one-to-one correlation between the peaks obtained from the resonance Raman spectrum compared to the SERRS spectrum, however there were frequency and intensity changes for some 20 peaks indicating interaction between the flavin and the silver particles. The activity of the enzyme while adsorbed to the nanoparticle surface was measured to be $86 \%$ of its natural activity which recovered to a $95 \%$ activity level when displaced from the surface. ${ }^{30}$

25 Broderick et al. have also shown the retention of enzyme activity for chlorocatechol dioxygenase on citrate reduced silver colloid, the activity remaining within the $60-85 \%$ range. ${ }^{31}$ They also demonstrated the sensitivity of SERRS for probing enzyme-substrate, enzyme-intermediates and enzyme30 product complexes leading to structural information during catalytic turnover.

Work by Rospendowski et al. clearly states the need for careful control of the biocompatibility of the silver colloid surface. ${ }^{32}$ Citrate reduced silver colloid was used, whose 35 protein compatibility was altered by selection of appropriate particle size and $\mathrm{pH}$ conditions. The activity of the enzymes, cytochromes P-450s, could be monitored in-situ by SERRS due to spectral variations arising from the conversion of the heme group from low to high spin upon addition of suitable 40 substrates. However, use of citrate reduced silver colloids is not suitable for all enzymes or even for all cytochromes. Bonifacio et al. have shown that structural changes in the active site are observed for cytochrome P450-2D6 with citrate reduced silver colloid but when treated with a 45 mercaptoalkanoic acid monolayer the enzyme retains its native structure when adsorbed to the colloid. ${ }^{33}$ The selfassembled monolayer (SAM) prevents direct contact between the enzymeand the nanoparticle surface and can be modified to give specific surface properties. Bjerneld et al. pretreated 50 their silver colloid with hydrogen peroxide which ensured the horseradish peroxidase under investigation retained $85 \%$ of its activity, compared to $50 \%$ when adsorbed directly onto citrate reduced silver colloid.

Obviously there are a large number of SAMs that could 55 potentially be used in such studies and a powerful technique which can screen the compatibility of a particular SAM with a specific enzyme is SERR-spectroelectrochemical analysis.
This system allows the redox ability of suitable enzymes to be probed by control of the electrode potential. Retention of the 60 activity can be proven and spectral variations relating to the enzyme state, which is a function of the controllable potential, can be monitored. ${ }^{34-36}$

After the enhancing medium has been chosen and optimised for biocompatiblity, $\mathrm{SE}(\mathrm{R}) \mathrm{RS}$ can be used to investigate the 65 activity of an enzyme towards different substrates or activators. Spectral changes between substrate-free and substrate-bound recombinant $\mathrm{P} 450_{\mathrm{BS} \beta}$ with silver colloid can be used to identify active substrates for the enzyme. ${ }^{37}$ Mantelingu et al. used SERS to investigate the activation of 70 the histone acetyltransferase activity of p300 by various analogues of ( $N$-(4-chloro-3-trifluoromethyl-phenyl)-2ethoxy-6-pentadecyl-benzamide) (CTPB). ${ }^{38}$ Structural changes seen within the SERS spectra were correlated to the enzyme activity indicating that this approach holds significant 75 promise for screening enzyme modulators for therapeutic applications. Enzyme-drug interactions have also been probed by SERS and by selection of a suitable excitation wavelength, interference from the enzyme and drug in the bulk solution. as well as adsorbed drug, could be limited. ${ }^{39,40}$

80 Although direct SE(R)RS of enzymes and their complexes in pure solutions is possible, when a new enzyme system is studied for the firdt time the biocompatibility of the enhancing substrate must be checked and optimised. Even after optimisation the enzyme activity can be suppressed. ${ }_{85}$ Competitive binding considerations further complicate the recording of direct enzyme $\mathrm{SE}(\mathrm{R}) \mathrm{RS}$ as the substrate, product or drug may have a greater affinity for the enhancing medium, displacing the enzyme. Therefore, direct enzyme SE(R)RS, although a very useful tool for elucidating enzyme structure 90 and interactions, cannot be used for in vivo work or for multiplex library screening where there are a myriad of biological molecules that can prevent the enzyme from binding to the surface. Therefore, an alternative approach for measuring enzyme activity by SERRS is required.

\section{${ }_{95}$ SERRS of an enzymatically produced dye}

Rohr et al. were the first to apply SERRS to immunoassays by attaching a resonant dye label, $p$-dimethylaminoazobenzene, to a thyroid stimulating hormone antibody. ${ }^{41}$ The immunoassay was carried out using a silver surface to 100 generate the SERRS signal. Significantly, using SERRS, which is a surface specific technique, as the preferred readout route removed the requirement of washing steps in the assay which is normally a time consuming stage in traditional ELISA experiments.

105 While Rohr et al. used dye labelled antibodies in their system, work by Dou et al. used the ability of specific enzymes to convert substrates into SERRS active dyes to produce a SERRS based sandwich ELISA for mouse-IgG. ${ }^{23}$ In this study, the secondary antibody was labelled with 110 peroxidase.. On addition of $o$-phenylenediamine and hydrogen peroxide, the peroxidase enzyme activity resulted in convertion to azoaniline, a SERRS active dye, with an absorption maximum centred at $420 \mathrm{~nm}$ (Figure 1). The concentrations of the enzyme, substrate and $\mathrm{H}_{2} \mathrm{O}_{2}$ used in the 
assay were low enough that they gave no detectable signal in contrast to the dye product, which gave a large SERRS signal.

The final reaction step within the ELISA (the rection between the antibody bound enzyme with the enzyme 5 substrate) was allowed to proceed for 20 minutes. After this time, $30 \mu \mathrm{l}$ of the reaction product was removed and the SERRS signal was obtained using sodium borohydride reduced silver colloid and $514.5 \mathrm{~nm}$ laser exciatation. This wavelength is significantly removed from the absorption 10 maximum of the dye and the colloid, 420 and $390 \mathrm{~nm}$ respectively, so the signal is likely to be non-resonant SERS. However, a linear relationship exists with respect to the intensity of the most significant dye peak, the $\mathrm{N}=\mathrm{N}$ stretching mode at $1442 \mathrm{~cm}^{-1}$ and the amount of complexed enzyme 15 modified antibody. Therefore, the concentration of the antigen of interest could be measured. The limit-of-detection was found to be $1.8 \times 10^{-15} \mathrm{~mol} / \mathrm{mL}$, an order of magnitude greater than the original work of Rohr et al. and confirmed later by work carried out by Zhao et al. ${ }^{42}$ This is mainly due to the fact 20 that the system does not rely on the measurement of covalently bound antibody-reporter dye complexes. The range over which the ELISA could be read linearly was narrow, $0.158-2.5 \mathrm{ng} / \mathrm{mL}$, however, this could likely be expanded by careful choice of the enzyme reaction time as well as the 25 colloid and substrate concentrations chosen for SERRS. Benefits of this system over traditional ELISA readout techniques included; only the enzyme product showed a strong SERRS spectrum and the strong fluorescent background was significantly reduced.

30 Stevenson et al. have applied SERRS to a standard horseradish peroxidase (HRP) ELISA system for the detection of human prostate specific antigen (PSA) within human serum, ${ }^{43}$ PSA being a valuable biomarker for the detection of prostate cancer. The substrate used in this ELISA was 2,2'35 azino-bis(3-ethylbenzothiazoline-6-sulfonic acid) (ABTS), which upon reaction with HRP and hydrogen peroxide formed a green oxidized product which had an absorbance at 420 nm. ${ }^{44}$ Using a commercially available PSA ELISA kit with gold nanoparticles, Stevenson et al. were able to decrease the 40 limit-of-detection into the $\mathrm{pg} \mathrm{ml}^{-1}$ range. Which is well below the currently accepted decisive limit of $4 \mathrm{ng} \mathrm{ml}^{-1}$ of PSA in serum. ${ }^{45}$

Peroxidase modified antibodies have also been used for the detection of membrane-bound enzymes in vivo. Hawi et al. 45 have investigated prostaglandin $\mathrm{H}$ synthase-1 and -2 (PGHS-1 and -2 ) in healthy hepatocytes and hepatocellular carcinoma cells, PGHS-2 being highly expressed in certain cancers. ${ }^{46}$ In this method the membrane enzymes were first reacted with a primary antibody before the peroxidase labelled secondary 50 antibody was added. The reaction substrates, $O$ phenylenediamine and hydrogen peroxide, were added and the azoaniline dye allowed to develop for 15 minutes before being probed with citrate reduced silver colloid using $632.8 \mathrm{~nm}$ laser excitation, this was therefore SERS (since the absorbance 55 maxima of the dye is $420 \mathrm{~nm}$ ) and did not benefit from additional resonance enhancements. When PGHS-1 was investigated using the dye produced by the peroxidase, the spectral intensity at $1370 \mathrm{~cm}^{-1}$ of the dye was similar in normal and cancer cells. However, PGHS-2 showed a much 60 stronger signal from cancerous cells than normal cells, Figure 2. This work demonstrated the power of measuring the SERS signal of an enzymatically produced dye for the histological investigation of membrane-bound enzymes. However, this approach could be expanded further and is clearly not limited 65 to membrane-bound enzymes, but rather any membrane species.

The work desribed above made use of standard ELISA methods in microwell plates where the enzyme reaction product was monitored in-situ by addition of metallic colloid. 70 However, work by $\mathrm{Wu}$ et al. has shown the ability to couple a bienzyme reaction pathway directly to gold colloid. ${ }^{47}$ The aim of this work was to measure glucose levels by SERRS, which was achieved by modifying gold colloidal nanoparticles with glucose oxidase (GOD) and horseradish peroxidase (HRP) 75 with the modified nanoparticles mixed with $o$-phenylenediamine and the glucose solution. Modification was achieved by simple mixing of the acidic gold colloidal nanoparticles with the enzyme solutions as has been reported before. ${ }^{48}$ The glucose was oxidized by the GOD to produce 80 hydrogen peroxide which was then combined with the $o$-phenylenediamine and converted to azoaniline by HRP. The azoanaline was then detected by SERRS, Figure 3 .

SERRS of the resulting azoaniline product could be used to measure the enzyme activity with respect to temperature, the 85 classical graph of activity vs. temperature being achieved with maximum activity observed at $40^{\circ} \mathrm{C}$, before decreasing due to denaturation of the enzymes. The resultant detection range was $0.50-32 \mathrm{mM}$ of glucose, which covers the average blood glucose range of 3.5-6.1 mM, and was six times wider than 90 previous literature values based on electrochemical glucose detection. $^{49}$

The work discussed so far in this section has been concerned with the use of enzymatically labelled antibodies, or colloidal nanoparticles, to produce a dye which can be 95 quantified and therefore used to infer the concentration of an antigen or another species, e.g. glucose. This approach, while reliant on the enzymes ability to produce a SERRS active dye, is not a measure of the enzyme directly or of its activity.. However, Ruan et al. have used the ability of alkaline 100 phosphatase to produce a SERRS active dye to detect the presence of the enzyme and monitor its activity. ${ }^{50}$

5-Bromo-4-chloro-3-indolyl phosphate (BCIP) is the substrate used for alkaline phosphatase (ALP), which hydrolyses the phosphate moiety of the BCIP and creates an 105 enol intermediate which is then oxidized to an insoluble blue BCIP dimer which is strongly SERRS active. The native BCIP gives no discernable spectral features, Figure 4. A standard linear response concentration profile of ALP was recorded between $4.1 \times 10^{-11}$ and $4.1 \times 10^{-15} \mathrm{M}$ after one hour of 110 incubation of the enzyme with the substrate. This limit-ofdetection was similar to or better than standard fluorescence assay techniques but benefited from a simpler experimental procedure and reduced analysis times. ${ }^{50}$

As with the work previously mentioned in this section, ALP 115 and its reaction product the insoluble blue BCIP dimer can be used to detect secondary species such as $\mathrm{C}$-reactive protein 
(CRP) as demonstrated by Campbell et al. ${ }^{51}$ Alkaline phosphatase conjugated anti-CRP antibody was added to an ELISA which was allowed to react with BCIP for 30 minutes before analysis with gold colloid. The CRP limit-of-detection 5 was found to be $0.3 \mathrm{ng} \mathrm{ml}^{-1}$ with a dynamic range up to 100 $\mathrm{ng} \mathrm{ml^{-1 }}$.

Experiments which monitor enzymatically produced dyes show a clear 'switching on' of the SERRS signal upon production of the dye. However, except in the work reported 10 by Ruan et al., the production of the dye by the enzyme was an indirect way to measure the amount of another species, be that another enzyme or an antigen, and as such is a general experimental readout technique for bioassays. Direct measurement of enzyme activity by SERRS has been achieved 15 by the application of masked dyes which show off-on activity in the presence of a suitable enzyme.

\section{SERRS of Enzymatically unmasked dyes}

Figure 5 shows the fundamental process behind the principle of using an enzyme to unmask a modified dye molecule. A 20 dye is modified with a chemical 'mask' which also renders it SERRS inactive as the mask prevents binding to the metal surface. However, the mask is designed such that it is enzyme cleavable in a specific manner. Once unmasked, the surface seeking group of the dye can now attach to the metallic 25 nanoparticle and produce a SERRS spectrum. The SERRS spectra obtained from the dyes is simpler than that obtained by non-resonance enhanced SERS since the spectra will be dominated by strongly allowed resonance-enhanced peaks which are easily identifiable above the other constituents 30 within the solution under analysis. This approach was first reported by Moore et al. who investigated hydrolases at ultralow concentrations using masked benzotriazole azo dyes. ${ }^{52}$

Significant work has been carried out to show that benzotriazole-based dyes are ideal compounds for adsorbing 35 onto the surface of metallic nanoparticles, in particular silver nanoparticles prepared by a citrate reduction method. ${ }^{53-55}$ The binding of the benzotriazole group displaces the citrate surface layer and generates a SERRS signal from the dye modified benzotriazole group. This binding affinity is greater 40 than that shown by molecules that naturally occur in biological fluids which significantly minimises potential interference. ${ }^{52}$

The benzotriazole group was used as the surface seeking group, therefore in the parent form it must be masked to 45 prevent interaction with the silver surface. Two components were placed onto the benzotriazole group, the first was a linker which could be cleaved by the action of a suitable enzyme. Further masking was provided by the inclusion of a specific enzyme recognition site which could either be the 50 enzyme substrate or a close analogue.

Moore et al. focussed on lipases and showed that the parent masked benzotriazole-dye complex gave a very low background signal (the off state) when compared to the free dye. The lipase from Pseudomonas cepacia was chosen due to 55 its ability to hydrolyse esters of 3-phenyl-butyric acid. Masked dye was mixed with a pre-aggregated solution of silver colloid, SERRS was recorded and the enzyme added.
The SERRS signal was observed to increase with time as the enzyme turned over the substrate, Figure 6 . The rate of ${ }_{60}$ hydrolysis was easily calculated from the resulting peak intensity vs. time graph.

An important consideration with this method is the specificity of the enzyme for the substrate which was shown by measuring the rate of enzyme activity for a range of 65 hydrolases which produced results which were consistent with the enzymes different reactivity towards the substrate. This was the first instance of SERRS being used as a tool to measure different enzyme activities. The speed of analysis was good with accumulation times of between 1 and 15 70 seconds, indicating the great promise for enzyme screening. Extrapolating an accumulation time of just one second per data point in a time course experiment shows there is the potential to screen $\sim 10^{5}$ enzymes per day, per instrument for activity towards a range of different substrates.

75 The stereoselectivity of the enzymes was measured by control of the chirality of the 3-phenylbutyric acid enzyme recognition site. Many of the lipase enzymes investigated were shown to hydrolyse the $S$-enantiomer faster than the $R$ enantiomer. However, for one enzyme this trend was reversed 80 and for others there was no significant rate difference between the two enantiomers (Figure 7). ${ }^{52}$ This stereoselectivity was observed to be consistent at all concentration ranges investigated and stereoselective rate data correlated with measurements carried out by standard HPLC techniques.

${ }_{85}$ The sensitivity of the technique was also probed by reacting $0.025 \mu \mathrm{g} / \mathrm{ml}$ of enzyme with the masked substrate for 600 seconds which corresponded to a detectable signal from only 0.8 picomoles of enzyme in the sample volume. When considering the interrogation volume versus the sample 90 volume it becomes obvious that single enzyme molecule detection is a possibility with this approach. It should be noted that the maximum product concentration, i.e. the concentration of the unmasked dye, which could be probed was $10^{-7} \mathrm{~mol} \mathrm{dm}^{-3}$, this was a result of signal saturation as all 95 available enhancing spots on the colloidal surface became coated.

Subsequent to this, the properties of the aryl azo benzotriazolyl alkyl esters have been further optimised and refined. Ingram et al. investigated the different enzyme 100 activities obtained using the $1 H / 3 H$ and $2 H$ isomers of the masked bezotriazole dye as well as using nucleophilic substitution of a chloromethyl ester by benzotriazolyl dyes which resulted in a ten-fold improvement with respect to the achieved synthetic yield for this particular isomer. ${ }^{56}$

105 Control of the isomerism of the masked benzotriazole was critical as it has been observed that the $1 \mathrm{H} / 3 \mathrm{H}$ isomers do not give as good a masking effect as the $2 H$ isomer. It has been suggested that this is a result of either steric hindrance consideration or electronic factors. Therefore, it was desirable 110 to use a synthetic method that selectively produced the $2 \mathrm{H}$ isomer in a higher yield. When the reaction of the sodium benzotriazolate with the chloromethyl ester was performed in acetone and anhydrous conditions the resultant isomer ratios were found to be $1: 2.5$ for $2 H$ and combined $1 H+3 H$. This 115 was significantly more than the trace amounts of the $2 H$ 
isomer which had been reported previously during the synthesis of the $1 H$ isomer and had to be purified with chiral HPLC. ${ }^{56}$

Due to the predominance of known chemistry for the 5 modification of $1 \mathrm{H}$ compounds it would be beneficial if these isomers could be masked to the same extent as the $2 \mathrm{H}$ isomers. This was achieved by Ingram et al. by adding an electron withdrawing group onto the aromatic ring of the benzotriazole, however, this required the synthetic route to be 10 modified further. The chloromethyl ester was converted to the iodomethyl analogue before the reaction was carried out in DMF rather than acetone. This resulted in no $2 \mathrm{H}$ isomer being observed, however a $20 \%$ yield of the $3 \mathrm{H}$ and a similar yield for the $1 H$ isomer was obtained. Significantly, these dyes 15 showed a masked SERRS activity comparable to the $2 H$ isomers due to the reduced basicity of the triazole ring which reduces the binding of these molecules to the silver colloidal surface. This route was the first report of the $1 H$ isomers being essentially SERRS inactive, which clearly results in the 20 potential lowering of the limit-of-detection for the technique when these dyes are used due to reduced background signals. ${ }^{56}$

The limit-of-detection of masked SERRS dyes was further improved by Ingram et al. when they synthesised masked 825 hydroxyquinolinyl azo dyes. ${ }^{57}$ This resulted in a sensitivity improvement of 100-fold when compared to the previous benzotriazole dyes. 8-Hydroxyquinoline will strongly chelate to a silver surface and its aryl azo derivatives are superb SERRS dyes. $o$-Acylation of the 8-hydroxy moiety prevented 30 the metal surface binding activity of the molecule, creating a masked dye which could be released by the action of an enzyme.

These probes are synthetically simpler to prepare due to the lack of potential isomerism and the reduced number of 35 synthetic steps. Incorporation of the SERRS active species was via a one step reaction from readily available starting materials, while the masking group was introduced via esterification using HATU. When compared to benzotriazole dyes, the initial hydrolysis rate with the lipase from ${ }_{40}$ Pseudomonas cepacia, was found to be greater due to the fact that phenolic ester hydrolysis is faster than the alternative alkyl ester hydrolysis. This led to a decrease in the limit-of-detection of the enzyme after a 30 minute reaction time to $\sim 0.2 \mathrm{ng} \mathrm{mL}^{-1}$, compared to a LoD of between 2 and 20 $45 \mathrm{ng} \mathrm{mL}^{-1}$ for the benzotriazole dye. Use of 8-hydroxyquinolinyl azo dyes allowed a faster accumulation of data with a lower limit-of-detection being achieved quicker than with masked benzotriazole dyes.

The initial work carried out using the experimental 50 approach of using specifically designed masked SERRS dyes concentrated almost exclusively on the action of lipases. In 2008, Ingram et al. reported the application of the technique to the detection of proteases. ${ }^{58}$ Over 500 genes encode for proteases which either non-specifically hydrolyse proteins or 55 carry out selective, efficient cleavage of specific substrates. ${ }^{59}$ Phenylalanine was attached to a dye labelled benzotriazole SERRS active species via a chloromethyl ester linker. This masked species gave no detectable SERRS spectra at a concentration of $10^{-7} \mathrm{M}$ until exposed to Subtilisin carlsberg 60 which cleaved the linker and freed the benzotriazole azo dye generating a SERRS signal.

Enantioselectivity of the protease was also demonstrated as no SERRS signal was generated when the experiment was repeated with the $D$-boc-phenylalanine enantiomer. This was 65 expected since the enzyme should show enantiospecificity for the natural substrate, therefore, the $D$ isomer was available to act as a negative control. Different rates of hydrolysis were observed when the protease enzyme was varied, similar to that previously observed for lipases. The substrate was selective 70 for proteases and was not unmasked in the presence of lipases. A signal could be observed from just $50 \mathrm{ng} \mathrm{ml}^{-1}$ of Subtilisin carlsberg after reaction with the masked substrate for 30 minutes. It is important to note that the accumulation time used to record the SERRS signal was only 1 second indicating 75 rapid analysis was easily achievable.

As well as providing impressive sensitivity, SERRS offers the ability to analyse multiple enzymes simultaneously and without the need for separation, which is of considerable benefit for multiplexed analysis. The multiplexing ability of 80 masked dyes was demonstrated in 2009 when phosphorylated and galactosylated analogues of the previous 8hydroxyquinolinyl azo dyes were prepared, Figure $8 \mathrm{a} .{ }^{60}$ This allowed the detection of alkaline phosphatase and $\beta$ galactosidase in one reaction mixture. Both enzymes have 85 slightly different $\mathrm{pH}$ operating windows, 8-9 and 6-8 respectively, therefore, all reactions were carried out at $\mathrm{pH} 8$ in a Tris buffer. The SERRS spectra of the different dyes provided Raman bands that could be used for differentiation, in particular, the $\mathrm{C}-\mathrm{H}$ bending present at $1267 \mathrm{~cm}^{-1}$ and the ${ }_{90} \mathrm{~N}=\mathrm{N}$-aryl stretching positioned at $1340 \mathrm{~cm}^{-1}$ identified the $\beta$-galactosidase and alkaline phosphatase respectively (Figure $8 b)$.

The majority of the previous described assay work focussed on the secondary quantification of another biological species ${ }_{95}$ i.e through binding of an antigen to a labelled antibody. The use of masked dyes which can be released by the action of an enzyme clearly demonstrates that SERRS can be used to probe the activity of an enzyme directly. Relative activity rates, enantioselectivity and enzyme selectivity have all been 100 demonstrated with this approach. Indeed with this multicomponent approach it can be applied to a wide range of potential enzymes and enzyme classes. The ability to alter the dye component attached to the surface seeking group such that they give distinct SERRS spectra has been demonstrated for 105 the identification of two enzymes in a mixture and suggests great promise for the development of greater multiplexing.

\section{Future Directions}

There are three clear directions for continued development of SERRS for monitoring enzymatic transformations. The first of 110 these areas is in vivo detection of enzymes and their activity. The method developed by Moore et al. and Ingram et al. is clearly the most suitable direction to develop. In vivo levels of enzyme detection have been shown as well as the ability to monitor relative enzyme activities and selectivities.

115 For all in vivo work a significant challenge is overcoming 
interference from other biological species within the cell. However, use of the surface seeking groups which are exposed upon enzyme action will ensure that interference from other species is minimised. The selection rules for ${ }_{5}$ SERRS also contribute to the rejection of interference as the most intense signals will be generated from species that meet the entire selection criterion and since these have been designed into the substrate, it will be the substrate that generates the most intense signal. A challenge still remains to 10 introduce the SERRS enhancing medium, metallic nanoparticles, and the masked substrate into the cells before measuring the enzyme activity. However, in vivo measurement of enzyme activity will provide significant levels of information about spatial locations and natural 15 activity (it is important to stress that the enzyme is not being labelled or modified in any respect with this approach and therefore this technique probes the natural activity of the enzyme).

An important feature of the masked substrate technique is 20 the ability to produce a wide range of substrates that vary with respect to the enzyme recognition site as well as the linked SERRS dye. This ability to build in flexibility within the substrate allows the potential for multiplexing, which has been demonstrated in the case of simultaneous detection of 25 alkaline phosphatase and galactosidase by SERRS which used different dye labels. ${ }^{60}$ This multiplexing ability can either be applied to in vivo studies or for the analysis of enzymatic libraries.

There has been a shift away from fundamental 30 understanding of enzyme structure-function considerations, towards the goal of making diversity screening an experimental reality. ${ }^{61}$ Enzyme libraries have been generated through directed evolution in an effort to improve an enzymes' specificity, selectivity or operational stability. ${ }^{62}$

35 This work is driven by the need for better enzymes in the chemical, pharmaceutical and food industries. However, directed evolution generates vast amounts of mutants, $10^{4}-10^{7}$, that must be screened after each round of evolution. This creates a bottle-neck within the development of new enzymes 40 as only small fractions of the libraries are generally screened, $10^{3}$ out of $10^{5}-10^{7} .{ }^{62}$ It has been stated that screening methods can be further improved if an analytical technique can be developed which measures the signal produced from natural or unnatural substrates which do not produce colour or 45 fluorescence. ${ }^{62}$

Clearly, the method of using enzyme specific masked dyes and SERRS would work well for the screening of new enzyme mutants. It is a rapid, inexpensive and easily automated technique. A real strength of this approach is the ability to so measure the enantioselectivity of an enzyme, which is essential particularly when the enzymes are being used as part of processes to produce pharmaceuticals.

Although the masked dye approach appears ideal for in vivo and large enzymatic library screening, there is one area where 55 an alternative approach will be required. This is in the single molecule detection (SMD) of an enzyme. Although reaction of a single enzyme with the masked substrate may produce an observable signal given a long enough incubation time, it will not provide any information about the enzyme modifications 60 during turnover.

The fundamental changes that an enzyme undergoes during turnover which results in the observed accelerated rates are still poorly understood ${ }^{5}$ although theory and computational investigations are shedding some light on this area, ${ }^{63}$ further ${ }_{65}$ experimental verification is required. Single molecule detection by SERRS has been demonstrated ${ }^{64-67}$ and $\mathrm{Xu}$ et al. have reported the SMD of haemoglobin by SERS. ${ }^{68}$ It would be particularly interesting if a SE(R)RS enzyme signal could be monitored on the millisecond-to-second timescale, which is 70 similar to that of enzymatic reactions. This would allow single enzyme kinetics to be recorded and may reveal kinetic data which is normally obscured by ensemble-averaged studies.

However, there remains several obstacles which need to be overcome before this can be achieved. These include the 75 development of a suitable enhancing substrate system which can bind a single enzyme without denaturing it as well as providing the required enhancement fields. Another more fundamental issue concerns the generated signals and what they mean. One proof that has been proposed for SMD is the 80 fluctuations observed in the spectra. ${ }^{10,69,70}$ Peak position, shape and relative intensity changes have all been observed. Some of these effects are most likely due to the solution based setup where molecules can adsorb and desorb from the 'hot spot', however, variations are still observed when the 85 molecules are confined to a surface. ${ }^{71-75}$ Therefore, the cause of spectral fluctuations must be fully analysed before SMD of an enzyme by SERRS can be properly explained otherwise it would not be clear if the variations within the spectra are due to enzyme modifications or non-ensemble effects.

\section{${ }_{90}$ Conclusions}

In this review we have focussed on the development of SERRS for the detection of enzyme transformations. Initially SE(R)RS was used as a means to detect enzymes, their substrates, products and inhibitors directly within pure 95 solutions. This involved the development of biocompatible colloids and careful experimentation to confirm that the addition of the enzyme onto the surface of a colloid did not alter its activity significantly as it could not be predicted $a$ priori. Once this was confirmed the signal variation could be 100 used to follow the bulk binding of an enzyme to it's substrate. However, all experiments reported some loss of activity when enzymes were combined with colloids.

Fortunately, the product of the reaction of several peroxidases and phosphatases with particular substrates 105 produce a dye molecule. This dye can then be detected by SERRS, which is an indirect measurement of the enzyme activity. This approach has been applied to the detection of secondary species, such as an antigen, by means of an immunoassay where the final step was to add a peroxidase 110 modified antibody to the assay and react the enzyme with the appropriate substrate to create the dye. Although this approach has the benefit of minimising signal interference within an immunoassay, it has limited applicability for the detection of a wide range of enzymes.

115 An approach capable of application to a wide range of 
enzymes and which measures the enzyme activity directly is the use of masked dyes which generate a SERRS signal upon enzyme action. The inherent modularity within this approach opens up the possibility of extensive multiplexing. Substrates 5 can be designed which are sensitive, selective and highlight enantioselectivity.

We have outlined the main challenges that still exist within this exciting field. Firstly, taking the masked dye technique and applying it for in vivo analysis. Secondly, analysis of 10 mutant enzyme libraries for increased speed of screening and thus improving the generation of novel enzymes for industrial applications and finally, the development of single molecule SERRS for the measurement of a single enzyme molecule during turnover. These are not insignificant challenges but the 15 important strides that have already been made to develop SERRS for the detection of enzymes and their activity provide a strong foundation from which these challenges can be met.

\section{Notes and references}

Centre for Molecular Nanometrology, WestCHEM, Department of Pure 20 and Applied Chemistry, University of Strathclyde, 295 Cathedral Street,

Glasgow,UK.E-mail: duncan.graham@strath.ac.uk; Tel: +44 141548 4701

1 D. Whitford Proteins; Structure and Function; John Wiley \& Sons Ltd.: Chichester, 2005.

252 L. Hedstrom, Chem. Rev., 2002, 102, 4429-4430.

3 K. A. Johnson, J. Biol. Chem., 2008, 283, 26297-26301.

4 K. H. Tang and M. D. Tsai, J. Cell. Physiol., 2008, 216, 315-320.

5 S. J. Benkovic and S. Hammes-Schiffer, Science, 2003, 301, 11961202.

306 R. Sharma, Y. Chisti and U. C. Banerjee, Biotechnolo. Adv., 2001, 19, 627-662.

7 M. Fleischmann; P. J. Hendra and A. J. McQuillan, Chem. Phys. Lett., 1974, 26, 163-166.

8 M. Moskovits, J. Raman Spectrosc., 2005, 36, 485-496.

359 E. C. Le Ru and P. G. Etchegoin Principles of Surface-Enhanced Raman Spectroscopy and related plasmonic effects.; Elsevier: Amsterdam, 2009.

10 K. Kneipp; Y. Wang; H. Kneipp; L. T. Perelman; I. Itzkan; R. Dasari and M. S. Feld, Phys. Rev. Lett., 1997, 78, 1667-1670.

4011 S. M. Nie and S. R. Emery, Science, 1997, 275, 1102-1106.

12 G. A. Baker and D. S. Moore, Anal. Bioanal. Chem., 2005, 382, 1751-1770.

13 S. Lal; N. K. Grady; J. Kundu; C. S. Levin; J. B. Lassiter and N. J. Halas, Chem. Soc. Rev., 2008, 37, 898-911.

4514 M. J. Banholzer; J. E. Millstone; L. D. Qin and C. A. Mirkin, Chem. Soc. Rev., 2008, 37, 885-897.

15 R. M. Jarvis and R. Goodacre, Chem. Soc. Rev., 2008, 37, 931-936.

16 I. Chourpa; F. H. Lei; P. Dubois; M. Manfait and G. D. Sockalingum, Chem. Soc. Rev., 2008, 37, 993-1000.

5017 N. P. W. Pieczonka and R. F. Aroca, Chem. Soc. Rev., 2008, 37, 946954.

18 D. Graham; K. Faulds and W. E. Smith, Chem. Comm., 2006, 43634371.

19 M. Moskovits, J. Chem. Phys., 1982, 77, 4408-4416.

5520 K. Faulds; R. Jarvis; W. E. Smith; D. Graham and R. Goodacre, Analyst, 2008, 133, 1505-1512.

21 K. Faulds; F. McKenzie; W. E. Smith and D. Graham, Angew. Chem. Int. Ed., 2007, 46, 1829-1831.

22 A. Macaskill; A. A. Chernonosov; V. V. Koval; E. A. Lukyanets; O.

${ }_{60}$ S. Fedorova; W. E. Smith; K. Faulds and D. Graham, Nucl. Acids Res., 2007, 35, e42.

23 X. Dou; T. Takama; Y. Yamaguchi; H. Yamamoto and Y. Ozaki, Anal. Chem., 1997, 69, 1492-1495.

24 L. A. Dick; A. J. Haes and R. P. Van Duyne, J. Phys. Chem. B., $65 \quad 2000,104,11752-11762$.
25 Y. J. Xiao; Y. F. Chen; T. Wang and X. X. Gao, Langmuir, 1998, 14, $7420-7426$.

26 Z. C. Yue; F. F. Zhuang; R. Kumar; L. Wang; S. B. Cronin and Y. H. Liu, Spectroc. Acta Pt. A-Molec. Biomolec. Spectr., 2009, 73, 226230.

27 Y.-J. Xiao; T. Wang; X.-Q. Wang and X.-X. Gao, J. Electroanal. Chem., 1997, 433, 49-56.

28 Y.-J. Xiao and J. P. Markwell, Langmuir, 1997, 13, 7068-7074.

29 Y. J. Xiao; X. X. Gao and J. Markwell, J. Electroanal. Chem., 1999, 465, 187-194.

30 R. A. Copeland; S. P. A. Fodor and T. G. Spiro, J. Am. Chem. Soc., 1984, 106, 3872-3874.

31 J. B. Broderick; M. J. Natan; T. V. Ohalloran and R. P. Vanduyne, Biochemistry, 1993, 32, 13771-13776.

8032 B. N. Rospendowski; K. Kelly; C. R. Wolf and W. E. Smith, J. Am. Chem. Soc., 1991, 113, 1217-1225.

33 A. Bonifacio; P. H. J. Keizers; N. P. E. Vermeulen; J. N. M. Commandeur; C. Gooijer and G. van der Zwan, Langmuir, 2007, 23, 1860-1866.

8534 S. Todorovic; A. Verissimo; N. Wisitruangsakul; I. Zebger; P. Hildebrandt; M. M. Pereira; M. Teixeira and D. H. Murgida, J. Phys. Chem. B, 2008, 112, 16952-16959.

35 J. Hrabakova; K. Ataka; J. Heberle; P. Hildebrandt and D. H. Murgida, Phys. Chem. Chem. Phys., 2006, 8, 759-766.

9036 A. Bonifacio; D. Millo; P. H. J. Keizers; R. Boegschoten; J. N. M. Commandeur; N. P. E. Vermeulen; C. Gooijer and G. van der Zwan, J. Biol. Inorg. Chem., 2008, 13, 85-96.

37 E. Bailo; L. Fruk; C. M. Niemeyer and V. Deckert, Anal. Bioanal. Chem., 2009, 394, 1797-1801.

9538 K. Mantelingu; A. H. Kishore; K. Balasubramanyam; G. V. P. Kumar; M. Altaf; S. N. Swamy; R. Selvi; C. Das; C. Narayana; K. S. Rangappa and T. K. Kundu, J. Phys. Chem. B, 2007, 111, 4527-4534.

39 A. Murza; S. Sanchez-Cortes and J. V. Garcia-Ramos, Biopolymers, 2001, 62, 85-94

10040 H. Morjani; J. F. Riou; I. Nabiev; F. Lavelle and M. Manfait, Cancer Res., 1993, 53, 4784-4790.

41 T. E. Rohr; T. Cotton; N. Fan and P. J. Tarcha, Anal. Biochem., 1989, 182, 388-398.

42 H. Y. Zhao; N. Yi; J. Wei; P. P. Luo; M. Z. Huang; G. Z. Yin and X. 105 M. Dou, Sci. China Ser. B-Chem., 2005, 48, 240-245.

43 R. Stevenson; A. Ingram; H. Leung; D. C. McMillan and D. Graham, Analyst, 2009, 134, 842-844.

44 K.-S. Shin and Y.-J. Lee, Arch. Biochem. Biophys., 2000, 384, 109115 .

11045 I. M. Thompson; D. K. Pauler; P. J. Goodman; C. M. Tangen; M. S. Lucia; H. L. Parnes; L. M. Minasian; L. G. Ford; S. M. Lippman; E. D. Crawford; J. J. Crowley and C. A. Coltman, Jr., N. Engl. J. Med., 2004, 350, 2239-2246.

46 S. R. Hawi; S. Rochanakij; F. Adar; W. B. Campbell and K. Nithipatikom, Anal. Biochem., 1998, 259, 212-217.

47 Z. S. Wu; G. Z. Zhou; J. H. Jiang; G. L. Shen and R. Q. Yu, Talanta, 2006, 70, 533-539.

48 A. Gole; C. Dash; C. Soman; S. R. Sainkar; M. Rao and M. Sastry, Bioconjugate Chem., 2001, 12, 684-690.

12049 G. Cui; J. H. Yoo; B. W. Woo; S. S. Kim; G. S. Cha and H. Nam, Talanta, 2001, 54, 1105-1111.

50 C. M. Ruan; W. Wang and B. H. Gu, Anal. Chem., 2006, 78, 33793384.

51 F. M. Campbell; A. Ingram; P. Monaghan; J. Cooper; N. Sattar; P. D. 125 Eckersall and D. Graham, Analyst, 2008, 133, 1355-1357.

52 B. D. Moore; L. Stevenson; A. Watt; S. Flitsch; N. J. Turner; C. Cassidy and D. Graham, Nat. Biotechnol., 2004, 22, 1133-1138.

53 G. McAnally; C. McLaughlin; R. Brown; D. C. Robson; K. Faulds; D. R. Tackley; W. E. Smith and D. Graham, Analyst, 2002, 127, 838841.

54 C. J. McHugh; F. T. Docherty; D. Graham and W. E. Smith, Analyst, 2004, 129, 69-72.

55 A. Enright; L. Fruk; A. Grondin; C. J. McHugh; W. E. Smith and D. Graham, Analyst, 2004, 129, 975-978.

13556 A. M. Ingram; K. Stirling; K. Faulds; B. D. Moore and D. Graham, Org. Biomol. Chem., 2006, 4, 2869-2873. 
57 A. Ingram; R. J. Stokes; J. Redden; K. Gibson; B. Moore; K. Faulds and D. Graham, Anal. Chem., 2007, 79, 8578-8583.

58 A. Ingram; L. Byers; K. Faulds; B. D. Moore and D. Graham, J. Am. Chem. Soc., 2008, 130, 11846-11847.

559 International-Human-Genome-Sequencing-Consortium, Nature, 2001, 409, 860-921.

60 A. Ingram; B. D. Moore and D. Graham, Bioorg. Med. Chem. Lett., 2009, 19, 1569-1571.

61 D. Wahler and J.-L. Reymond, Curr. Opin. Biotechnol., 2001, 12, 535-544.

62 N. Cohen; S. Abramov; Y. Dror and A. Freeman, Trends Biotechnol., 2001, 19, 507-510.

63 M. Garcia-Viloca; J. Gao; M. Karplus and D. G. Truhlar, Science, 2004, 303, 186-195.

1564 J. A. Dieringer; K. L. Wustholz; D. J. Masiello; J. P. Camden; S. L. Kleinman; G. C. Schatz and R. P. Van Duyne, J. Am. Chem. Soc., 2009, 131, 849-854.

65 E. C. Le Ru; M. Meyer and P. G. Etchegoin, J. Phys. Chem. B, 2006, 110, 1944-1948.

2066 N. P. W. Pieczonka; G. Moula and R. F. Aroca, Langmuir, 2009, 25, 11261-11264.

67 J. A. Dieringer; R. B. Lettan; K. A. Scheidt and R. P. Van Duyne, J. Am. Chem. Soc., 2007, 129, 16249-16256.

68 H. Xu; E. J. Bjerneld; M. Käll and L. Börjesson, Phys. Rev. Lett., 1999, 83, 4357 - 4360.

69 K. Kneipp; H. Kneipp; G. Deinum; I. Itzkan; R. R. Dasari and M. S. Feld, Appl. Spectrosc., 1998, 52, 175-178.

70 Z. H. Zhou; G. Y. Wang and Z. Z. Xu, Appl. Phys. Lett., 2006, 88,

71 N. P. W. Pieczonka and R. F. Aroca, ChemPhysChem, 2005, 6, $2473-$ 2484.

72 A. Otto, J. Raman Spectrosc., 2002, 33, 593-598.

73 P. G. Etchegoin; M. Meyer; E. Blackie and E. C. Le Ru, Anal. Chem., 2007, 79, 8411-8415.

74 P. G. Etchegoin; M. Meyer and E. C. Le Ru, PCCP, 2007, 9, 3006353010.

75 C. J. L. Constantino; T. Lemma; P. A. Antunes and R. Aroca, Anal. Chem., 2001, 73, 3674-3678.

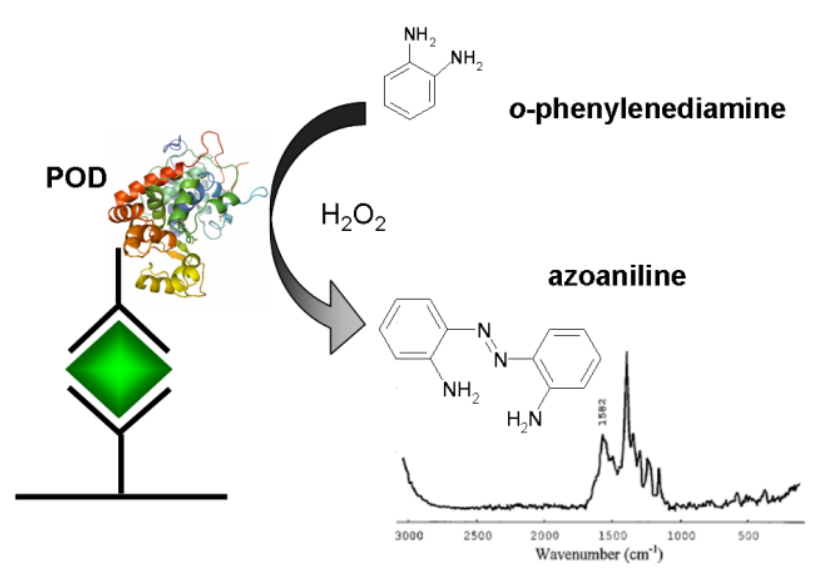

Fig. 1. SERS enzyme immunoassay system with SERS spectra of the resulting enzymatically produced dye. (Modified from ref. 23)

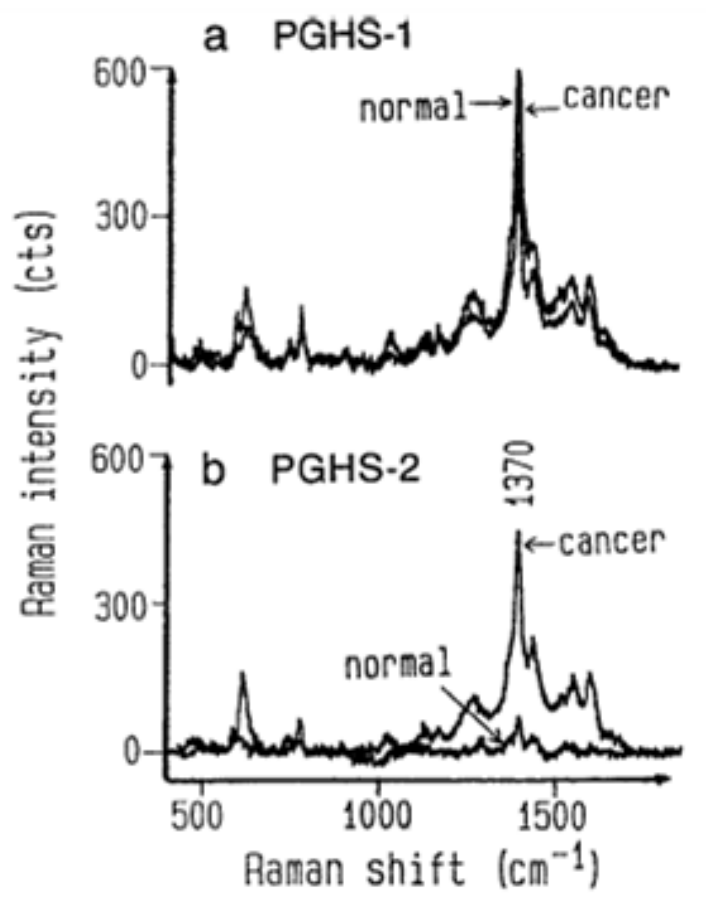

Fig. 2. (a) SERS spectra of PGHS-1 in normal and malignant human hepatocytes; (b) SERS spectra of PGHS-2 in normal hepatocytes and malignant cells. (Reproduced from ref. 46)

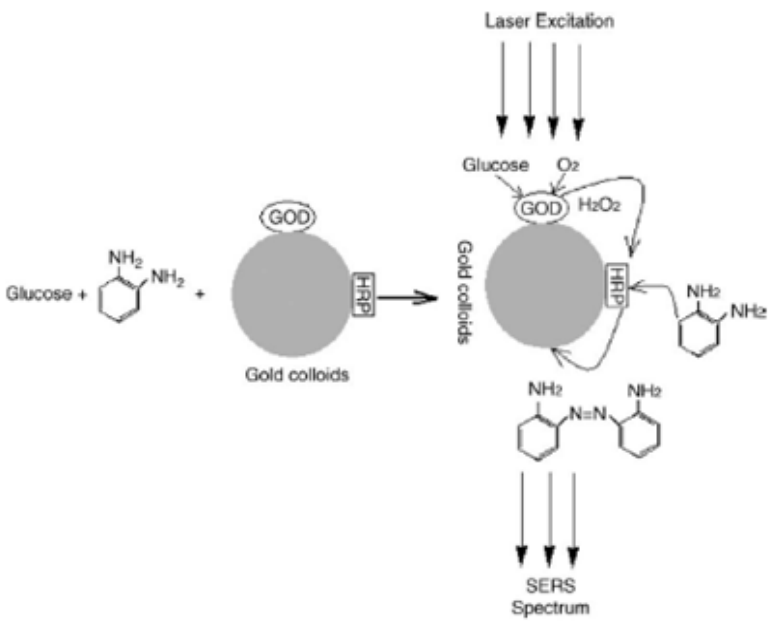

Fig. 3. Oxidation of $o$-phenylenediamines catalyzed by GOD/HRP bienzymatic system and the principle of the indirect detection of glucose. 50 
a

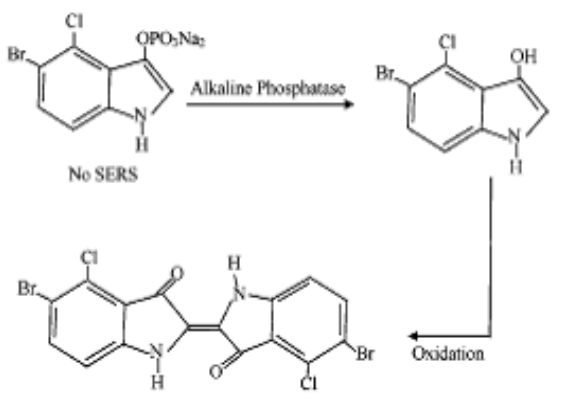

Indigo dye SERS

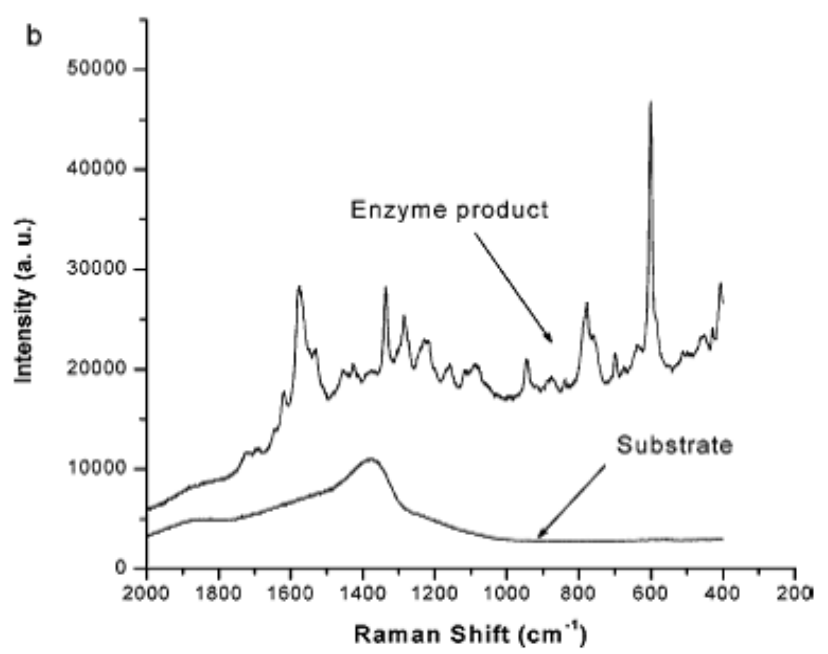

\section{Dye}
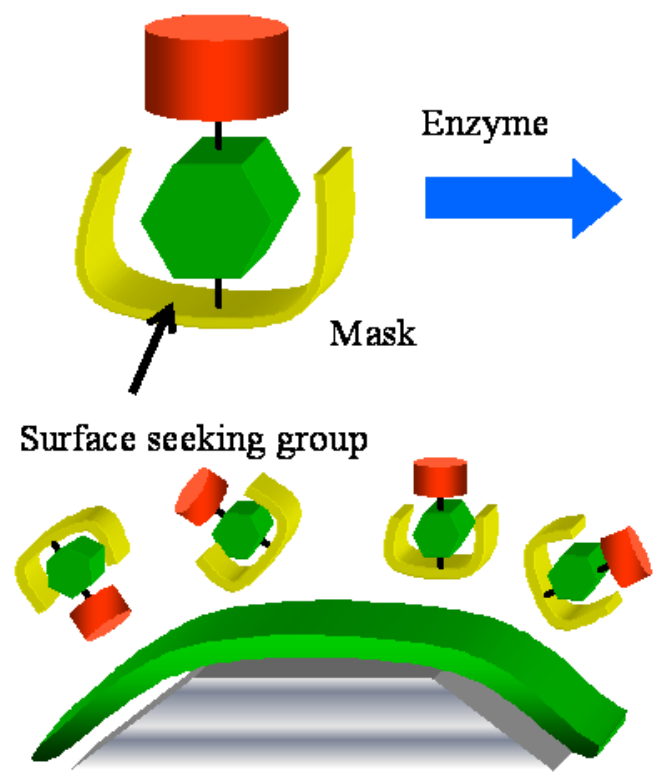

No SERRS
Fig. 4. (a) Structure of 5-bromo-4-chloro-3-indolyl phosphate and its enzymatic reaction products, BCIP dimer; (b) corresponding SERS spectra of BCIP (substrate) at $0.3 \mathrm{mg} / \mathrm{mL}$ and its reaction products. ALP concentration $10^{-10} \mathrm{M}$, reaction time 1 hour, $785 \mathrm{~nm}$ laser, $\sim 1.5 \mathrm{~mW}$, acquisition time $10 \mathrm{~s}$. (Reproduced from ref. 50)

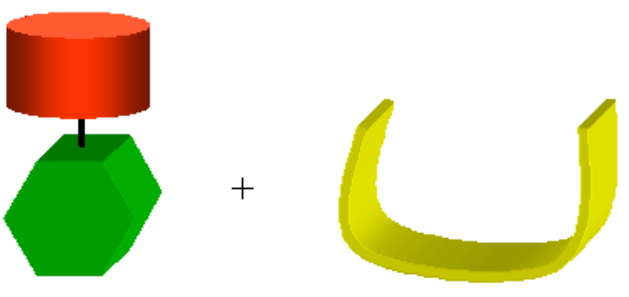

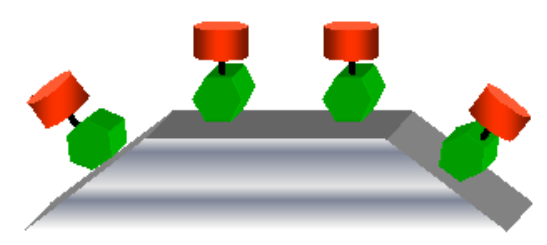

SERRS

Fig. 5. The principle of obtaining SERRS through the unmasking of a benzotriazole azo dye that is incapable of surface adsorption due to the presence of an enzyme substrate. Enzymatic hydrolysis of the bond linking the substrate to the dye releases the dye which then gives SERRS. (Reproduced from ref. 


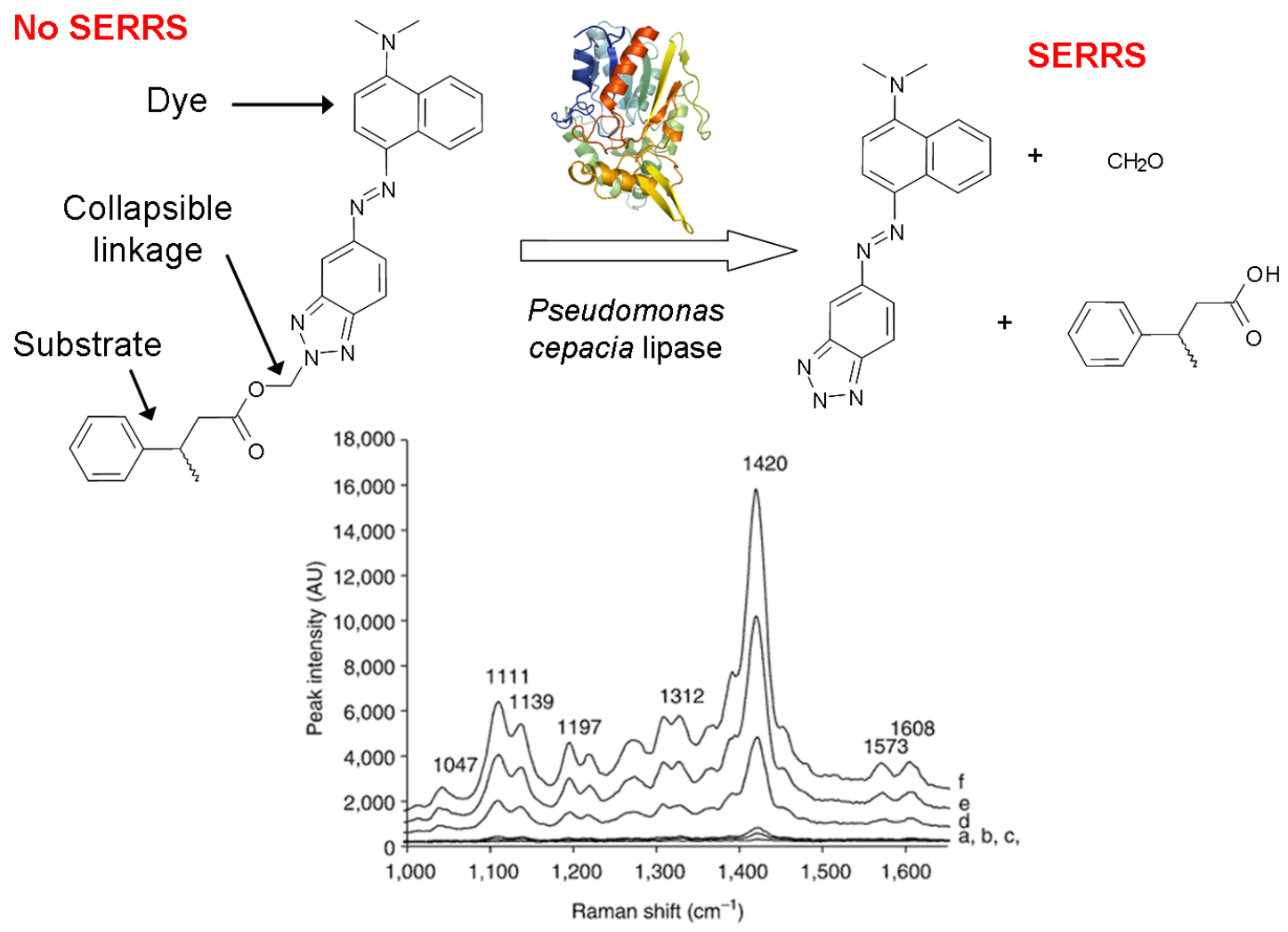

Fig. 6. SERRS spectra with time following lipase-catalyzed cleavage of the masked Napthalene-based azo-benzotriazole dye. Substrate was present at $1 \times 10^{-7} \mathrm{M}$, Pseudomonas cepacia lipase at a final concentration of $0.4 \mathrm{mg} / \mathrm{ml}$ was added after $200 \mathrm{~s}$ and silver nanoparticles were activated with poly(Llysine). Spectra were accumulated for $1 \mathrm{~s}$ using $514.5-\mathrm{nm}$ excitation through a microscope objective into a standard cuvette. Five replicate measurements 5 were taken and the average value used to plot the graph. Spectra accumulated from 1,800-800 cm-1 . a, $0 \mathrm{~s} ; \mathrm{b}, 100 \mathrm{~s} ; \mathrm{c}, 200 \mathrm{~s} ; \mathrm{d}, 400 \mathrm{~s} ; \mathrm{e}, 800 \mathrm{~s} ; \mathrm{f}, 1600 \mathrm{~s}$. (Modified from ref. 52)
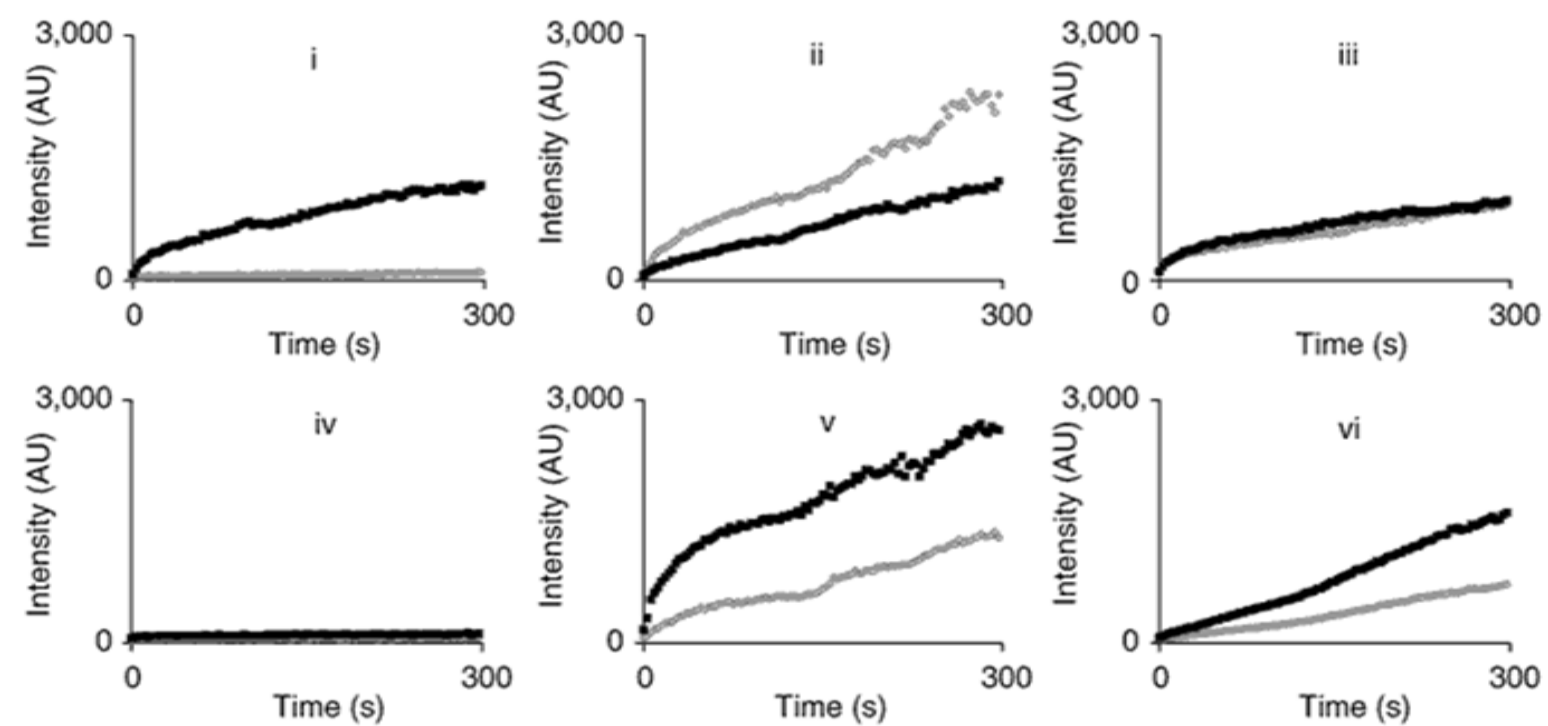

Fig. 7. The enzyme-catalyzed hydrolysis of the S- and R-enantiomers of the napthalene-based azo-benzotriazole dye substrate were monitored, dark trace, 10 S-enantiomer; light trace, R-enantiomer. Concentrations were $1.4 \mu \mathrm{g} / \mathrm{ml}$ enzyme and $7.1 \times 10^{-7} \mathrm{M}$ substrate. Enzymes used: i, Alcaligenes spp. lipase; ii, Candida cylindracea lipase; iii, Candida rugosa esterase; iv, Penicillium camemberti lipase; v, Pseudomonas stutzeri lipase; vi, Rhizopus oryzae lipase. (Reproduced from ref. 52) 
a)

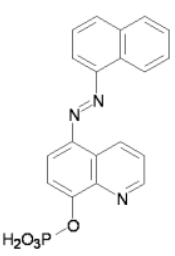

Masked dye substrate for

alkaline phosphatase

b)
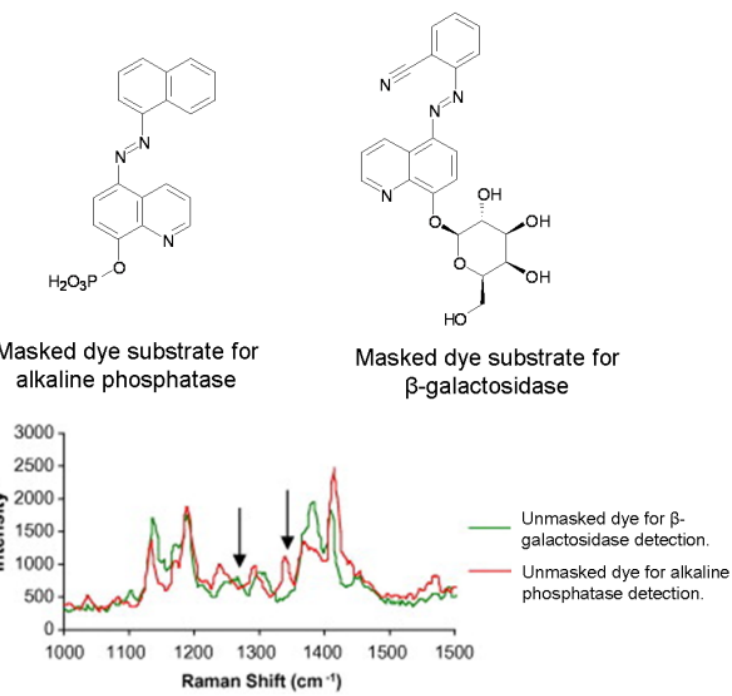

Masked dye substrate for

$\beta$-galactosidase

Fig. 8. (a) Structures of the masked dye substrates for the detection of alkaline phosphatase and $\beta$-galactosidase; (b) superimposed SERRS spectra for unmasked dyes, peaks used for discrimination, at $1267 \mathrm{~cm}^{-1}$ and $1340 \mathrm{~cm}^{-1}$ are highlighted. (Modified from ref. 60) 